\title{
A SEARCH FOR FEATURES IN EARLY-TYPE GALAXIES ${ }^{a)}$
}

\author{
KATE EBNETER \\ Astronomy Department, University of California, Berkeley, California 94720
}

S. DJoRgovsKI

Harvard-Smithsonian Center for Astrophysics, 60 Garden Street, Cambridge, Massachusetts 02138

\author{
MARC DAVIS \\ Astronomy Department, University of California, Berkeley, California 94720 \\ Received 22 May 1987; revised 7 October 1987
}

\begin{abstract}
We have conducted a search for dust lanes, incipient stellar disks, bars, shells, and other deviations from elliptical symmetry in a sample of 159 early-type galaxies. The data are from the CCD surface-photometry survey of Djorgovski (1985a), for which the selection effects are well understood. The imageprocessing technique used is division by a purely elliptical model image, constructed from the surfacephotometry profiles for a given object (best-fit surface brightness, ellipticity, and position angle as functions of semimajor axis). Our sample contains 116 elliptical galaxies, 33 SO galaxies, and ten intermediate types. Some galaxies that might better be classified as "dusty ellipticals" (in that they contain no obvious stellar disk) are included among the S0s. Forty-two of the ellipticals (36\%) either definitely or very likely contain dust, either in patches or in well-defined lanes or rings. Five of the E/S0 galaxies $(50 \%)$ also show possible or definite dust, as do $15(47 \%)$ of the S0s. Three of the elliptical galaxies definitely contain stellar disks, with several more possible candidates. Thus, approximately $50 \%$ of the elliptical galaxies show "features" of some kind. The detection of features is dependent on the signal-to-noise ratio of the data, so these numbers represent lower limits. The presence of dust in the galaxies classified as ellipticals does not seem to depend on any intrinsic characteristic of the galaxy. The dusty ellipticals do seem to prefer low-density environments. The "elliptical" galaxies with disks tend to have higher ellipticities and larger two-wave Fourier residuals, an effect also described by Carter (1987). These galaxies may be preferentially found in higher-density environments, and may be lowerluminosity systems, but these statements are based on very small-number statistics (ten galaxies out of a total of 116), and no strong conclusions may be drawn. However, our results clearly further blur the distinction between ellipticals and S0s.
\end{abstract}

\section{INTRODUCTION}

The "classical" view of elliptical galaxies holds that they contain no dust and little gas, and no trace of a stellar disk of any kind. However, dust patches in ellipticals were reported by Hubble (1930), who admittedly did not recognize them as such. Any early-type galaxy found to contain such features was usually instantly classified as an S0 galaxy. This practice unfortunately tended to blur the distinction between galaxies that were flattened, rotationally supported systems without spiral arms (i.e., S0s) and those that were ellipsoidal, anisotropic pressure supported systems (i.e., genuine large ellipticals) containing significant amounts of gas and dust. There are, of course, many examples of genuine elliptical galaxies containing dust and neutral gas now known (Hawarden et al. 1981; Ebneter and Balick 1985; Sadler and Gerhard 1985; Sparks et al. 1985; Knapp, Turner, and Cuniffe 1985). Indeed, it seems that most ellipticals probably contain dust at some level (Jura 1982, 1986), although very few of them contain substantial disks of dust and gas like that found in NGC 5128 (Centaurus A), the well-known, but somewhat atypical "dusty" elliptical. Furthermore, Carter (1987) has recently shown that some rapidly rotating ellipticals contain weak stellar disks, and he suggests that such disks may be a common component of rapidly rotating ellipticals. It is important to determine how frequently dust, "incipient" stellar disks, and other features

a) Based in part on data obtained at Lick Observatory, University of California.

(e.g., shells, jets, etc.) occur in bona fide ellipticals, as such features clearly can provide important clues to the origin and evolution of both elliptical and disk galaxies. In particular, they may provide information on the frequency of galaxian mergers and galactic "cannibalism," and help to probe the intrinsic shapes of elliptical galaxies (see, for example, Merritt and de Zeeuw 1983).

It has also been suggested recently that mergers play an important role in the formation of powerful extragalactic radio sources, which are almost exclusively found in elliptical or elliptical-like galaxies (Heckman et al. 1986; Djorgovski et al. 1987). It is therefore very important to know the frequency with which features that may be signs of merging occur in an optically selected sample of elliptical and S0 galaxies, in order to provide a comparison sample for the radio ellipticals.

We have conducted a search for morphological features in a large sample of early-type galaxies, defining a "feature" as any deviation from elliptical symmetry in the underlying light. The data come from the CCD surface-photometry survey of Djorgovski (1985a), which covered $\sim 200$ elliptical and $\sim 50 \mathrm{~S} 0$ galaxies. Some of the images obtained by Djorgovski were found to suffer from defects which made them unsuitable for processing, as discussed further below. From the original sample of $\sim 250$ objects, we examined images of a total of 193 galaxies. Some of these images were then found not to be usable because of cosmetic defects or severe electronics noise, and our final sample consists of 159 galaxies, of which 116 had previously been classified as ellipticals, 33 
were classified as S0s, and ten were transitional types (E/ S0). (We do not necessarily agree with these classifications in all cases; see below.) An important aspect of our survey is that the completeness effects are well understood. Similar but smaller surveys have been conducted by Lauer (1985b) (using the same telescope, detector, and software) and Sparks et al. (1985); Gallagher (1986) also conducted a search for dark nebulae in a small number of elliptical galaxies using several different image-processing techniques.

\section{IMAGES AND IMAGE PROCESSING}

The images used in this search were obtained as part of an extensive CCD surface-photometry survey of elliptical and S0 galaxies. Detailed information on the sample selection, data acquisition, reduction, and calibration may be found in Djorgovski (1985a). All of the data were obtained with the $1 \mathrm{~m}$ Nickel telescope of Lick Observatory and either a TI $500 \times 500 \mathrm{CCD}$ with a Canon speed-up lens, or a GEC $576 \times 385$ CCD. The GEC CCD was cosmetically far superior to the TI device. The filter used for all of the observations was the "Spinrad red" filter described and calibrated by Djorgovski (1985b).

In most cases there were multiple images of each object, so the best image was selected whenever possible. The images to be used for the search were selected according to the following criteria:

(1) The longest exposure available was used. In the case of TI CCD images, only images with exposures of $300 \mathrm{~s}$ or longer were used, as shorter exposures could not in general be flatfielded adequately. (The solitary exception is a $100 \mathrm{~s}$ exposure of the bright central regions of M32.) This criterion eliminated five of the galaxies from the original sample.

(2) Where possible, GEC CCD images were preferred to TI CCD images.

(3) If two images were obtained on the same night, the second of the two was used, as the placement of the galaxy on the CCD was usually better, avoiding bad columns and other pixel defects. For some galaxies, both images were processed, but generally only one image was used.

(4) Images with notations that the image was trailed or poorly focused, or taken in bad seeing, were rejected unless no other image was available. In some cases the images were so bad that they could not be used at all; eight galaxies were eliminated from the original sample in this way.

(5) Some of the images taken with the TI CCD early in the project were not usable for our purposes because they could not be flatfielded adequately. This eliminated 20 galaxies from the original Djorgovski (1985a) sample.

(6) Some images for the surface-photometry survey were taken with a Nikon speed-up lens and the GEC CCD. These images suffered from rather severe distortions and were not used in this search. This eliminated an additional 19 galaxies from the Djorgovski (1985a) sample.

The images taken with the Lick GEC CCD were modeled using the surface-photometry profiles of the same, single image. Those taken with the Lick TI $500 \times 500$ CCD were modeled using average surface-photometry profiles taken from several images, wherever possible. This produced better results for these images, as the overall image quality was much poorer for this CCD. (We also modeled three GEC images - those of NGC 750, NGC 751, and NGC 4660 - with the average surface-photometry profiles. In the case of NGC 750 and NGC 751, there was no significant difference between the models obtained with the average profiles and those obtained with the single profiles. In the case of NGC 4660 , a disk was detected that was not unambiguously detected when the galaxy was modeled with a single profile. This result is discussed in more detail in Sec. III.)

After weeding out the images according to these criteria, we had 193 galaxy images, which were processed by the technique described below. The survey coverage in terms of apparent and absolute magnitudes is illustrated in Fig. 1. The absolute magnitudes are within the $r_{\mathrm{e}}$ elliptical isophotes (from a fit to the de Vaucouleurs profile), in the $r_{G}$ (Spinrad red GEC) bandpass, computed assuming a spherical, nonlinear Virgocentric infall model with Local Group infall velocity of $400 \mathrm{~km} \mathrm{~s}^{-1}$, and $H_{0}=100 \mathrm{~km} \mathrm{~s}^{-1} \mathrm{Mpc}^{-1}$ (see Djorgovski and Davis 1987 for details on how the absolute magnitudes are defined). The apparent magnitudes are taken from the CfA redshift survey (Huchra et al. 1983) and are rather heterogeneous; see that reference and Djorgovski (1985a) for a full discussion of the apparent magnitudes.

In some cases, the processed images were found to be unusable, for a variety of reasons. In all cases, if another suitable image of the galaxy was available, that image was processed. However, this was not always possible, and we were forced to eliminate such galaxies from the sample. We found, for

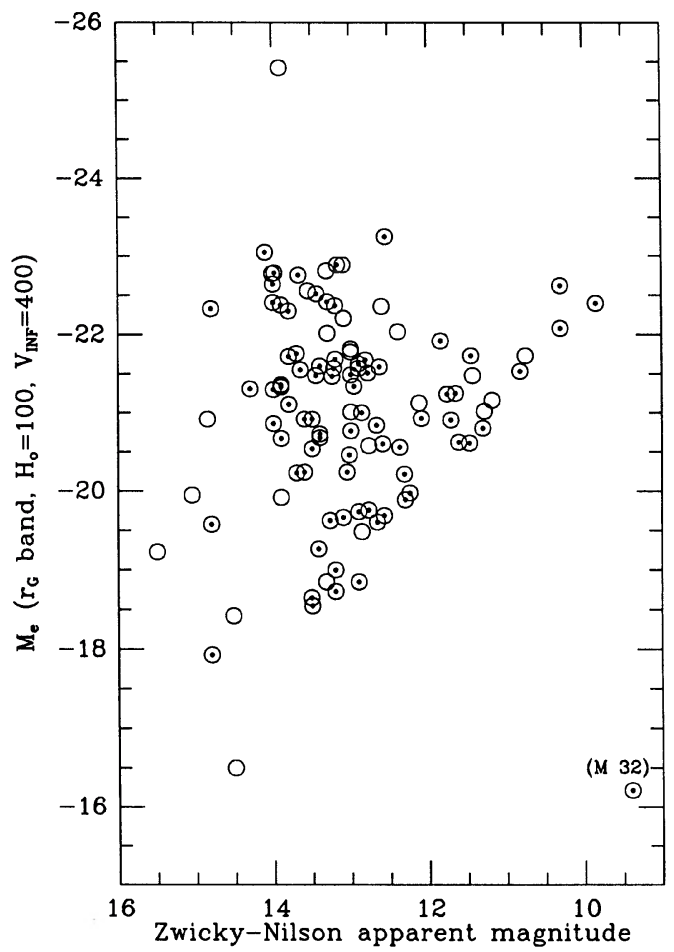

Fig. 1. Distribution of the survey galaxies in terms of apparent and absolute magnitudes. The circles represent all galaxies from the survey by Djorgovski (1985a) for which there is reliable distance information (and thus an absolute magnitude), and the circles with dots in them represent those galaxies examined for morphological features in this work. The absolute magnitudes are within the $r_{\mathrm{c}}$ elliptical isophotes (from a fit to the de Vaucouleurs profile), in the $r_{G}$ (red) bandpass, computed assuming a spherical, nonlinear Virgocentric infall model with Local Group infall velocity of $400 \mathrm{~km} \mathrm{~s}^{-1}$, and $H_{0}=100$ $\mathrm{km} \mathrm{s}^{-1} \mathrm{Mpc}^{-1}$ (see Djorgovski and Davis 1987 for details of how the absolute magnitudes are defined). 
instance, that close pairs of galaxies (where the two galaxies actually overlap in the image) were all but impossible to model properly. We rejected a total of seven galaxies from the sample for this reason. (In one case the companion was not in the original sample.) Four of the TI images were found to have been inadequately flatfielded, so that the only features visible in the residual image were due to (wellmapped) sensitivity variations on the CCD. For galaxies of small angular scale, the surface photometry was often not very good, and the results of the modeling, even with smoothing the profiles, were not useful; we rejected six images for this reason. On four more occasions the galaxies were just very poorly placed on the CCD with respect to bad pixels or bad columns. Sometimes things simply went wrong for reasons that we did not understand; we rejected at least seven images for these reasons. Finally, six images turned out to have severe periodic electronics noise or noise caused by the CCD "baseline" (bias), and had to be rejected. The total number of galaxies eliminated for such reasons was 34 , leaving us with 159 galaxies in the final survey. Because some of the unusable images were of galaxies of small angular size, there is some discrimination against such objects in the final sample. However, as Fig. 1 shows, this does not translate into any discrimination against intrinsically faint galaxies.

The principal problem involved in searching for features in elliptical or elliptical-like galaxies is suppressing the strong radial intensity gradient of the underlying light. One of the best techniques for accomplishing this is to divide the image of the galaxy by a perfectly elliptical model image. In this technique we take the surface photometry for a given object (in this case, best-fit surface brightness, ellipticity, and position angle as functions of semimajor axis), and construct a model galaxy image that obeys the measured profiles, but with perfectly elliptical isophotes. The program for doing the image reconstruction was written by Tod Lauer, and run under the image-processing program VISTA (Stover et al. 1985). Details of the modeling program are described by Lauer (1985a).

The surface-photometry fitting algorithm is described by Djorgovski (1985a); all of the profiles appear in that reference. The surface-photometry profiles were smoothed before making the model image, because the reconstruction routine follows the original profiles exactly, noise and all. For the surface-brightness profiles, the first 30 points in the profile (the central regions of the galaxy) were kept intact. The remainder of the profile was then median filtered with a 7 pixel window and then smoothed with a five-point boxcar smoothing. The ellipticity and position-angle profiles were first median filtered with a 7 pixel window. The first ten points were kept with linear spacing, and the remaining points were rebinned into logarithmic radial bins with a step size of 0.1 in $\log (r)$ (pixels). The spread of values within each radial bin was used as a weighting function. A fifthorder polynomial was then fit to the new points, and the profile was replaced by the polynomial-fit values. (For the P.A. profiles, the $180^{\circ}$ ambiguity was first removed from the profiles.) This filtering of the profiles is necessary, because it smooths over some of the residual pixel-to-pixel noise, and avoids creation of artifacts in processed images: for example, a spurious "glitch" in a surface-brightness profile would expand into a dark or luminous ring, and a small twist in ellipticity or P.A. profile near the nucleus can produce an artificial "dust lane." A glitch in the surface-brightness profile of sev- eral percent produces a comparable artificial feature in the residual. The effect of glitches in the P.A. and ellipticity profiles is harder to quantify. Only contours that were more than $80 \%$ complete in azimuthal coverage were used for the fits, as there was usually a great deal of noise associated with the less complete contours.

From these smoothed surface-brightness, ellipticity, and P.A. profiles, we then created a model image by two-dimensional interpolation. The center of each galaxy was located using a mirror-autocorrelation technique (Djorgovski 1987a). We used the same center for the model images as was used to generate the surface photometry in the first place. The original image was then divided by the model image, which has the same surface-brightness, ellipticity, and position-angle profiles as the original image, but purely elliptical, "noiseless" isophotes. The model may also be subtracted from the original image, but it is more difficult to display the results uniformly. The results are the same, however. Clearly, if there are no features in the galaxy, that is, no deviations from purely concentric, elliptical isophotes, the quotient image will have an average value of unity. (It would be exactly one if the original image had no noise in it.) This is the same technique as used by Lauer (1985b). Sparks et al. (1985) used a similar technique, except that they used images in two colors. They modeled the red image, and divided the blue image by the model red image, to produce a color map. This "two-color" technique is especially useful in searching for dust features, which are characteristically red, and it is superior to the similar technique used by Ebneter and Balick (1985) of simply dividing the blue image by the red image. Comparisons of various image-enhancement techniques are given by Djorgovski (1986) and Gallagher (1986).

The centering of the model image is clearly very important, as a small deviation of the center can cause artifacts in the rest of the quotient image. In most cases this was not a problem, but we have noted in Table I 11 cases in which the center was bad. (A bad center-even by a fraction of a pixel-causes a very noticeable "dipole" effect in the center of the galaxy, namely, a bright spot where the model image is too low and a dark spot where it is too high.) The centering may also be disturbed by the presence of dust near the center of the galaxy, as shown by NGC 5485 (see Fig. 3(c)). This produces a characteristic signature, however: a pair of "dipoles". Images showing this feature are listed in Table I as containing "possible" or "probable" dust unless the dust clearly shows in a lane; there are 23 such galaxies in Table I.

All of the quotient images were displayed on a TV display device with a uniform contrast level by displaying from $5 \%$ below the mean (nominally, one) to $5 \%$ above the mean. The images were then inspected by eye for features. The images were inspected by both K. E. and S. D., in most cases with no knowledge of previously identified features. This is admittedly a somewhat subjective process. However, some of the features that seemed to be right at the limits of detection have, in fact, been detected by others (such as the dust filament in NGC 5846 and the nuclear dust patch in NGC 1199), which gives us some confidence that the weak features we observe are indeed real (see the comments on individual objects, below). Examples of images with various types of morphological features, before and after the modelimage removal, are shown in Fig. 2. We note that the modelimage-removal technique is a powerful tool for investigations of dynamical resonances and other stellar features in 
TABLE I(a). Elliptical galaxies.

\begin{tabular}{|c|c|c|c|c|c|}
\hline ID & TYPE & FEATURES & CCD & COMMENTS & $\operatorname{EXP}(\mathrm{s})$ \\
\hline N 57 & $\mathbf{E}$ & Weak features? & GEC & Image trailed & 500 \\
\hline N 97 & & None & TI & & 500 \\
\hline N 185 & $\mathrm{dE} 3$ & Lots of dust & GEC & Poor image & 500 \\
\hline N 194 & $\mathbf{E}$ & Poss. weak dust & $\mathrm{TI}$ & & 500 \\
\hline N 221 & E2 & None & $\mathrm{TI}$ & Center saturated & 100 \\
\hline N 430 & $\mathrm{E}$ & None & TI & Image not very good & 600 \\
\hline N 507 & $\mathrm{E}$ & Dust lane in center & GEC & Wrong ID? & 500 \\
\hline N 533 & E3 & None & TI & & 500 \\
\hline N 596 & E0 & None & TI & & 500 \\
\hline N 636 & E1 & None & TI & Bad center & 500 \\
\hline N 680 & $\bar{E}$ & Poss. patchy dust & TI & & 300 \\
\hline N 720 & E5 & None & $\mathrm{TI}$ & Image rather poor & 500 \\
\hline N 741 & E0 & None & $\mathrm{TI}$ & Star near nucleus & 500 \\
\hline N 750 & E0 & None & GEC & Paired $\mathrm{w} / 751$ & 500 \\
\hline N 751 & $\overline{\mathbf{E}}$ & None & GEC & Paired w/750 & 500 \\
\hline N 821 & E6 & None & $\mathrm{TI}$ & Bad focus? & 500 \\
\hline N 855 & $\mathbf{E}$ & Poss. dust near nucl. & TI & Poor image, bad center & 500 \\
\hline N 1016 & $\mathrm{E}$ & None & TI & & 300 \\
\hline N 1132 & $\mathrm{E}$ & None & GEC & & 500 \\
\hline N 1199 & E2 & Weak dust in nucl. & $\mathrm{TI}$ & Previously reported & 500 \\
\hline N 1209 & E6 & None & TI & & 500 \\
\hline N 1395 & E2 & $\begin{array}{l}\text { Weak quad. features } \\
\text {-disk? }\end{array}$ & GEC & & 500 \\
\hline N 1439 & E1 & None & GEC & & 500 \\
\hline N 1453 & E2 & None & $\mathrm{TI}$ & Poor center & 500 \\
\hline N 1521 & E3 & None & GEC & & 500 \\
\hline N 1550 & & None & TI & & 500 \\
\hline N 1587 & & None & $\mathrm{TI}$ & Paired w/N1588 & 300 \\
\hline N 1588 & {$[\mathrm{E} 1 \mathrm{p}]$} & None & $\mathrm{TI}$ & $\begin{array}{l}\text { Paired w/N1587 } \\
\text { Bad center; “disturbed” }\end{array}$ & 300 \\
\hline N 1600 & E4 & $\begin{array}{l}\text { Dust patches near } \\
\text { nucleus }\end{array}$ & $\mathrm{TI}$ & & 300 \\
\hline N 2476 & $\mathrm{E} ?$ & $\begin{array}{l}\text { nucleus } \\
\text { Prob. irr. dust }\end{array}$ & GEC & & 500 \\
\hline N 2749 & $\mathrm{E}$ & None & GEC & Very bad seeing & 500 \\
\hline N 2865 & E4 & Prob. none & GEC & Prob. star on nucleus & 500 \\
\hline N 2924 & E0 & None & GEC & & 500 \\
\hline N 2943 & $\mathrm{E}$ & None & GEC & & 500 \\
\hline N 2986 & E2 & None & GEC & Bad center & 500 \\
\hline N 3070 & $\mathbf{E}$ & Dust patches & GEC & & 500 \\
\hline N 3091 & E3 & None & GEC & & 500 \\
\hline N 3193 & $\mathrm{E} 2$ & None & GEC & Bad center & 500 \\
\hline N 3209 & $\mathrm{E}$ & Prob. small dust patch & GEC & & 500 \\
\hline N 3325 & E: & None & GEC & & 500 \\
\hline N 3605 & $\mathrm{E} 5$ & Weak dust in nucleus & GEC & & 500 \\
\hline N 3608 & E1 & $\begin{array}{l}\text { Dust patches near } \\
\text { nucleus }\end{array}$ & GEC & Electronics noise & 500 \\
\hline
\end{tabular}


TABLE I(a). (continued)

\begin{tabular}{|c|c|c|c|c|c|}
\hline ID & TYPE & FEATURES & CCD & COMMENTS & $\operatorname{EXP}(\mathbf{s})$ \\
\hline $\begin{array}{l}\text { N } 3610 \\
\text { N } 3613 \\
\text { N } 3641\end{array}$ & $\begin{array}{l}\text { E5 } \\
\text { E6 } \\
\text { E }\end{array}$ & $\begin{array}{l}\text { Disk, boxy isophotes } \\
\text { Quad feature-disk? } \\
\text { None, or VERY weak } \\
\text { dust }\end{array}$ & $\begin{array}{l}\text { GEC } \\
\text { GEC } \\
\text { GEC }\end{array}$ & $\begin{array}{l}\text { Electronics noise } \\
\text { Bad center } \\
\text { Electronics noise }\end{array}$ & $\begin{array}{l}500 \\
500 \\
500\end{array}$ \\
\hline $\begin{array}{l}\text { N } 3818 \\
N 3853 \\
N 3862 \\
N 4168 \\
N 4439\end{array}$ & $\begin{array}{l}\mathrm{E} 5 \\
\mathrm{E} \\
\mathrm{E} \\
\mathrm{E} 2\end{array}$ & $\begin{array}{l}\text { Ring of dust? } \\
\text { Prob. none } \\
\text { Prob. none } \\
\text { Poss. dust near nucl. } \\
\text { None }\end{array}$ & $\begin{array}{l}\text { GEC } \\
\text { GEC } \\
\text { GEC } \\
\text { GEC } \\
\text { GEC }\end{array}$ & & $\begin{array}{l}500 \\
500 \\
500 \\
500 \\
500\end{array}$ \\
\hline N 4261 & E3 & Possible dust & GEC & $\begin{array}{l}\text { Reported dust; minor } \\
\text { axis rotation }\end{array}$ & 500 \\
\hline N 4278 & E1 & $\begin{array}{l}\text { Dust patches near } \\
\text { nucleus }\end{array}$ & GEC & Previously reported & 500 \\
\hline $\begin{array}{l}N 4318 \\
N 4360\end{array}$ & $\begin{array}{l}\mathrm{E} ? \\
\mathrm{E}\end{array}$ & $\begin{array}{l}\text { Poss. extr. weak dust } \\
\text { None }\end{array}$ & GEC & Bad center & $\begin{array}{l}500 \\
500\end{array}$ \\
\hline N 4374 & E1 & Strong dust lane & GEC & $\begin{array}{l}=\text { M84; well-known dust; } \\
\text { in Virgo cluster; } \pm 20 \%\end{array}$ & 500 \\
\hline N 4387 & $\mathrm{E}$ & $\begin{array}{l}\text { Prob. small dust patch } \\
\text { quad. feature-disk? }\end{array}$ & GEC & & 500 \\
\hline $\begin{array}{l}N 4478 \\
N 4486\end{array}$ & E0 & $\begin{array}{l}\text { None } \\
\text { Jet }\end{array}$ & GEC & Previously reported & $\begin{array}{l}500 \\
500\end{array}$ \\
\hline $\begin{array}{l}\text { N } 4489 \\
\text { N } 4551\end{array}$ & $\mathbf{E}$ & Poss. dust in nucl. & TI & & 300 \\
\hline $\begin{array}{l}\text { N } 4551 \\
\text { N } 4555\end{array}$ & $\begin{array}{l}\mathbf{E} \\
\mathbf{E}\end{array}$ & $\begin{array}{l}\text { Quad. ring-disk? } \\
\text { None }\end{array}$ & $\begin{array}{l}\text { GEC } \\
\text { GEC }\end{array}$ & & $\begin{array}{l}500 \\
500\end{array}$ \\
\hline N 4564 & E6 & Very messy; disk & GEC & Electronics noise & 500 \\
\hline N 4581 & $\mathbf{E}$ & Poss. very weak dust & GEC & & 500 \\
\hline $\begin{array}{l}N 4660 \\
N 4730\end{array}$ & E5 & Stellar disk & GEC & Disk is fairly small; $\pm 10 \%$ & 500 \\
\hline $\begin{array}{l}\text { N } 4739 \\
\text { N } 4742\end{array}$ & {$\left[\begin{array}{l}{[\mathrm{E} 1]} \\
\mathrm{E} 4\end{array}\right.$} & $\begin{array}{l}\text { None } \\
\text { Nucl. dust, shell? }\end{array}$ & $\begin{array}{l}\text { GEC } \\
\text { GEC }\end{array}$ & & 500 \\
\hline N 4760 & EO & None & GEC & Star near center & 500 \\
\hline N 4786 & {$[\mathrm{E} 3 \mathrm{p}]$} & None & GEC & Bad center & 500 \\
\hline N 4889 & & None & GEC & & 500 \\
\hline$N 4915$ & E0 & Prob. small dust patch & GEC & & 500 \\
\hline N 5198 & E1 & None & TI & & 500 \\
\hline N 5216 & [E0p] & Poss. dust in nucl. & TI & & 500 \\
\hline N 5322 & $\mathrm{E} 4$ & Shell? & GEC & Bad center & 500 \\
\hline N 5490 & $\mathrm{E}$ & None & GEC & Bad center & 500 \\
\hline N 5507 & $\mathrm{E}$ & Probable dust & GEC & So? & 500 \\
\hline N 5557 & E2 & Chaotic structure & GEC & S0 & 500 \\
\hline N 5576 & E4 & Boxy isophotes? & GEC & & 500 \\
\hline N 5582 & $\mathbf{E}$ & Prob. nucl. dust & GEC & & 500 \\
\hline N 5623 & $\mathbf{E}$ & Prob. patchy dust & GEC & & 500 \\
\hline N 5638 & E1 & $\begin{array}{l}\text { Poss. v. weak, } \\
\text { diffuse dust }\end{array}$ & GEC & & 500 \\
\hline
\end{tabular}


TABLE I(a). (continued)

\begin{tabular}{|c|c|c|c|c|c|}
\hline ID & TYPE & FEATURES & $\mathrm{CCD}$ & COMMENTS & $\operatorname{EXP}(s)$ \\
\hline N 5687 & E3 & Dust near nucleus & GEC & So? & 500 \\
\hline N 5796 & E1 pec & None & GEC & & 500 \\
\hline N 5812 & & Possible weak dust & GEC & Electronics noise & 500 \\
\hline N 5813 & E1 & Possible dust patches & GEC & & 500 \\
\hline N 5831 & E4 & $\begin{array}{l}\text { Prominent skew dust } \\
\text { lane }\end{array}$ & TI & $\pm 20 \%$ & 300 \\
\hline N 5845 & $\mathrm{E}$ & $\begin{array}{l}\text { Dust lane; small disk? } \\
\text { Slight quad. feature }\end{array}$ & GEC & & 500 \\
\hline N 5966 & $\mathrm{E}$ & None & $\mathrm{TI}$ & Bad center & 300 \\
\hline N 5982 & E3 & None & TI & Poor quality & 200 \\
\hline N 6051 & $\mathbf{E}$ & None & $\mathrm{TI}$ & & 300 \\
\hline N 6086 & $\mathrm{E}$ & None & TI & & 300 \\
\hline N 6127 & $\bar{E}$ & None & GEC & & 500 \\
\hline N 6137 & $\mathbf{E}$ & None & TI & & 300 \\
\hline N 6146 & $\mathrm{E}$ & None & GEC & & 500 \\
\hline N 6173 & $\mathbf{E}$ & Poss. dust lane & GEC & Weak quad. feature & 500 \\
\hline N 6482 & E2 & Poss. shells? & GEC & Star on nucleus & 500 \\
\hline $\begin{array}{l}\text { N } 6487 \\
\text { N } 7391\end{array}$ & $\begin{array}{l}\mathrm{E} \\
\mathrm{E}\end{array}$ & $\begin{array}{l}\text { None } \\
\text { Poss. dust lane near } \\
\text { nucl. }\end{array}$ & $\begin{array}{l}\text { GEC } \\
\text { GEC }\end{array}$ & & $\begin{array}{l}500 \\
500\end{array}$ \\
\hline N 7454 & $\mathrm{E}$ & Dust lane near nucl. & GEC & & 500 \\
\hline N 7458 & $\overline{\mathrm{E}}$ & $\begin{array}{l}\text { Boxy isophotes or } \\
\text { patchy dust? }\end{array}$ & GEC & & 500 \\
\hline N 7562 & $\mathbf{E}$ & None & GEC & Bad center, noisy & 500 \\
\hline N 7619 & E3 & None & GEC & Bad center & 500 \\
\hline N 7626 & E1 & None & GEC & & 500 \\
\hline N 7628 & $\mathrm{E}$ & $\begin{array}{l}\text { Weak dust patches } \\
\text { near center? }\end{array}$ & $\mathrm{TI}$ & & 500 \\
\hline N 7660 & $\mathrm{E}$ & $\begin{array}{l}\text { Poss. dust patch in } \\
\text { nucleus }\end{array}$ & $\mathrm{TI}$ & & 300 \\
\hline N 7768 & $\mathrm{E}$ & None & TI & Very noisy & 300 \\
\hline N 7778 & $\mathrm{E}$ & Poss. VERY weak dust & GEC & & 500 \\
\hline N 7785 & E5 & $\begin{array}{l}\text { Dust patches near } \\
\text { nucleus; poss. weak } \\
\text { disk? }\end{array}$ & GEC & Quad. feature & 500 \\
\hline I 179 & $\mathbf{E}$ & None & $\mathrm{TI}$ & & 500 \\
\hline I 745 & $\ldots$ & None & GEC & & 500 \\
\hline I 962 & $\ldots$ & None & GEC & & 500 \\
\hline I 1211 & $\mathbf{E}$ & None & GEC & & 500 \\
\hline I 4051 & $\mathrm{E}$ & None & GEC & & 1000 \\
\hline I 4562 & $\mathrm{E} ?$ & Poss. dust patch & GEC & & 500 \\
\hline $\begin{array}{l}\text { U } 10115 \\
\text { Mrk } 626\end{array}$ & $\begin{array}{l}\mathbf{E} \\
\mathbf{E}\end{array}$ & $\begin{array}{l}\text { None } \\
\text { None }\end{array}$ & $\begin{array}{l}\text { GEC } \\
\text { GEC }\end{array}$ & & $\begin{array}{l}500 \\
500\end{array}$ \\
\hline
\end{tabular}


TABLE I(b). S0 galaxies.

\begin{tabular}{|c|c|c|c|c|c|}
\hline ID & TYPE & FEATURES & $\mathrm{CCD}$ & COMMENTS & $\operatorname{EXP}(s)$ \\
\hline N 128 & So & Peanut shaped bulge & GEC & Well known; $\pm 20 \%$ & 500 \\
\hline N 474 & $\mathrm{RSO} / \mathrm{a}$ & Shells? & GEC & $=$ Arp 227 & 500 \\
\hline N 584 & & $\begin{array}{l}\text { Substantial stellar } \\
\text { disk }\end{array}$ & GEC & $\pm 20 \%$ & 500 \\
\hline N 670 & $\mathrm{Sb}:(?)$ & $\begin{array}{l}\text { Poss. patchy dust; } \\
\text { disk }\end{array}$ & GEC & So (UGC) & 500 \\
\hline N 890 & So & Ambiguous: disk? & GEC & Quad. feature & 500 \\
\hline N 936 & $\mathrm{SBO} / \mathrm{a}$ & Bar with ears & GEC & SB0 galaxy; $\pm 20 \%$ & 500 \\
\hline N 1172 & & Ambiguous & GEC & Chip defect on nucleus & 500 \\
\hline N 1638 & & Prob. dust patches & TI & E/So (UGC) & 500 \\
\hline N 4382 & So pec & Dust or disk?, tail? & $\mathrm{TI}$ & $=\mathrm{M} 85 ; \pm 10 \%$ & 500 \\
\hline N 4464 & [SO] & $\begin{array}{l}\text { Unlikely, but poss. } \\
\text { diffuse dust }\end{array}$ & GEC & Poor seeing, noisy & 500 \\
\hline N 4552 & So & Shells & GEC & = M89; elliptical? & 500 \\
\hline N 4649 & So & $\begin{array}{l}\text { Either weakly boxy, } \\
\text { or weak, diffuse, } \\
\text { patchy dust }\end{array}$ & GEC & $\begin{array}{l}=\mathrm{M} 60=\mathrm{VV} 206 ; \text { very } \\
\text { weak quad. feature }\end{array}$ & 500 \\
\hline N 4673 & S0: & None & GEC & Elliptical? & 500 \\
\hline N 5424 & so & Ambiguous & GEC & Elliptical? & 500 \\
\hline N 5485 & S0 pec & Very strong dust lane & GEC & Previously known; $\pm 20 \%$ & $\begin{array}{l}500 \\
500\end{array}$ \\
\hline $\begin{array}{l}\text { N } 5631 \\
\text { N } 5739\end{array}$ & $\begin{array}{l}\mathrm{S} 0 / \mathrm{Sa} \\
\mathrm{Sa}\end{array}$ & $\begin{array}{l}\text { Dust patch near nucl? } \\
\text { Prob. dust ring near } \\
\text { nucleus }\end{array}$ & $\begin{array}{l}\text { GEC } \\
\text { GEC }\end{array}$ & So/Sa (UGC) & $\begin{array}{l}500 \\
500\end{array}$ \\
\hline N 5838 & So & None & GEC & Boxy bulge? & 500 \\
\hline N 5846 & So & $\begin{array}{l}\text { Off-nuclear dust } \\
\text { "filament" }\end{array}$ & GEC & Noisy. UGC lists as E. & 50 \\
\hline N 5854 & $\mathrm{Sa}$ & Unusual-boxy? & GEC & So (UGC, RC2) & 500 \\
\hline N 5864 & $\mathrm{SBa}$ & Similar to N5854 & GEC & SBO (UGC, RC2) & 500 \\
\hline N 6278 & S0 & Dust patch near nucl. & GEC & & 50 \\
\hline N 6359 & & Dust patch near nucl. & GEC & & 50 \\
\hline N 6501 & $\mathrm{~S} 0 / \mathrm{Sa}$ & Poss. dust patch & GEC & Star near nucleus & $\begin{array}{l}50 \\
50\end{array}$ \\
\hline N 6548 & SB0 & Disk/bar? & TI & & 50 \\
\hline N 7280 & $\mathrm{SO} / \mathrm{Sa}$ & Dust near center & GEC & & $\begin{array}{l}50 \\
50\end{array}$ \\
\hline N 7377 & So/Sa pec & Patchy dust & GEC & & $\begin{array}{l}500 \\
500\end{array}$ \\
\hline N 7465 & SBO & $\begin{array}{l}\text { Lots of messy dust } \\
\text { in center }\end{array}$ & GEC & & 500 \\
\hline $\begin{array}{l}\text { N } 7585 \\
\text { N } 7634\end{array}$ & $\begin{array}{l}\text { So/Sa } \\
\text { SBO }\end{array}$ & $\begin{array}{l}\text { Very messy-tails, ? } \\
\text { Center very weird-- } \\
\text { dust, boxy isophotes? }\end{array}$ & $\begin{array}{l}\text { GEC } \\
\text { GEC }\end{array}$ & Arp 223 & $\begin{array}{l}500 \\
500\end{array}$ \\
\hline N 7836 & $\cdots$ & $\begin{array}{l}\text { Very chaotic dust } \\
\text { near nucleus }\end{array}$ & GEC & SB0, spiral? & 500 \\
\hline $\begin{array}{l}\text { I } 1153 \\
\text { A } 0659\end{array}$ & $\begin{array}{l}\text { S0 } \\
\text { S0 }\end{array}$ & $\begin{array}{l}\text { Prob. patchy dust } \\
\text { None }\end{array}$ & $\begin{array}{l}\text { GEC } \\
\text { TI }\end{array}$ & Anon. $0659+6505$ & $\begin{array}{l}500 \\
500\end{array}$ \\
\hline
\end{tabular}


TABLE I(c). E/S0 galaxies.

\begin{tabular}{llllll}
\hline \hline ID & TYPE & FEATURES & CCD & COMMENTS & EXP(s) \\
\hline N 205 & S0/E5 & Lots of dust patches & TI & Image quality poor & 300 \\
N 529 & E/S0 & Unlikely, but poss. & GEC & & 500 \\
N 1060 & E/S0 & Unlikely, but poss. & GEC & & 500 \\
N 1726 & E4/S0? & Poss. dust patches & TI & & 500 \\
N 4472 & E1/S0 & Dust lane? Funny.. & GEC & $=$ M49 & 500 \\
N 4636 & E0/S0 & None & GEC & & 500 \\
N 4692 & E/S0 & Poss. weak dust & GEC & & 500 \\
N 5423 & E/S0 & Poss. weak dust patch & GEC & & 500 \\
N 5493 & E7/S0 & Boxy, asymmetric disk & GEC & Prob. S0 & 500 \\
N 7052 & E/S0? & None & GEC & E/S0 classification uncertain. & 500 \\
& & & & Quad. feature-S0? & \\
\hline \hline
\end{tabular}

disk galaxies, as illustrated here with the example of the SB0 galaxy NGC 936 (Figs. 2(c), 2(d)). Several other processed images are shown in Fig. 3, and are briefly discussed below.

The results of the survey are summarized in Table I. As noted above, some of the 193 images that were processed turned out in the end to be too noisy for use or to have other problems that precluded any useful findings. These images are not included in Table $\mathbf{I}$ or in our statistics. The term "quad. feature" in Table I indicates the presence of a feature resembling a "quadrupole," that is, a ring of alternating dark and bright features. Such a feature can be seen in the processed image of NGC 936 (Fig. 2(d)). Galaxies showing this type of feature were reprocessed, sometimes by redoing the surface photometry, paying very close attention to the location of the center for the isophotes. In many such cases (most notably those of NGC 584 and NGC 4660, see below) the result was the appearance of a definite stellar disk. In some cases, however, the quadrupole feature persisted even with improved profiles. We believe that such features are caused by the presence of a disk, which makes the total isophotes non-elliptical and causes a numerical instability in the surface-photometry algorithm. Better-quality data should be obtained on these galaxies to determine if they do indeed possess disks, or weakly "boxy" isophotes.

The Hubble types of the galaxies are taken, in order of preference, from the Revised Shapley-Ames Catalog of Bright Galaxies (Sandage and Tammann 1981, hereafter referred to as the RSA), the Second Reference Catalog of Bright Galaxies (de Vaucouleurs, de Vaucouleurs, and Corwin 1979, hereafter referred to as the RC2), and the Uppsala General Catalog of Galaxies (Nilson 1973, hereafter referred to as the UGC). In some instances we have indicated alternate classifications where these sources disagree.

\section{COMMENTS ON INDIVIDUAL GALAXIES}

NGC 584. This galaxy is variously classified as an elliptical or an S0. The processed image clearly shows a prominent stellar disk. (The smaller, tilted disk in the nuclear region may or may not be real.) This disk was previously reported by Lauer (1985b) and Djorgovski and Ebneter (1986); its presence was also inferred by Carter (1987). The galaxy is a rapid rotator (Davies and Illingworth 1983). Our first processed image showed the so-called "quadrupole" feature dis- cussed in Sec. II; inspection of the surface-photometry profiles showed that there were "notches" in the ellipticity and position-angle profiles, apparently caused by some type of numerical instability in the surface-photometry algorithm in the presence of very non-elliptical isophotes. Careful rereduction of the data allowed us to generate a set of profiles without the "notches," and the resultant image shows the very obvious disk of the galaxy (Fig. 2(b)). Note that the disk is not at all apparent in the unprocessed image of the galaxy (Fig. 2(a)).

NGC 936. This SB0 galaxy has been studied in some detail by Kormendy (1983). We include it here (Figs. 2(c), 2(d)) mainly to illustrate the power of the present method of image enhancement. Note the "ears" on the ends of the bar, which are not readily apparent in the original image.

NGC 4278. Gunn (1978) reported that there were dust patches in the nuclear regions of this gas-rich, mildly active elliptical (Faber et al. 1978; Knapp, Kerr, and Williams 1978). It was observed by Gallagher (1986), who did not detect any dark features. Our image (Fig. 3(a)) shows faint dust patches near the center of the galaxy, confirming Gunn's report.

NGC 4374. The dust lane in this Virgo elliptical (Figs. $2(\mathrm{e}), 2(\mathrm{f}))$ was discovered by Wade (1960), and is well known. The axis of the dust lane is skewed with respect to the isophotes of the stellar component of the galaxy. The galaxy is the host of an extended, double-lobed radio source (3C 272.1 ). The radio ejection axis is essentially perpendicular to the dust lane (Kotanyi and Ekers 1979). Forman, Jones, and Tucker (1985) have observed a corona of x-ray-emitting gas around NGC 4374; no neutral hydrogen has been observed in the galaxy (Ebneter and Balick, unpublished data; van Gorkom, private communication), but it was detected by IRAS. In these respects it is similar to NGC 1316 (Fornax A), which also contains substantial amounts of dust and has an X-ray corona, but no detectable $\mathrm{H} \mathrm{I}$ (Ebneter and Balick, unpublished data; van Gorkom, private communication ).

NGC 4660. Like NGC 584, this galaxy contains a rather prominent stellar disk, which first appeared as a quadrupole feature. In this instance, the average surface-photometry profiles generated by Djorgovski (1985a) were used to make a second model and quotient image, which showed the presence of the disk (Fig. 3(b)). This disk was not previously 


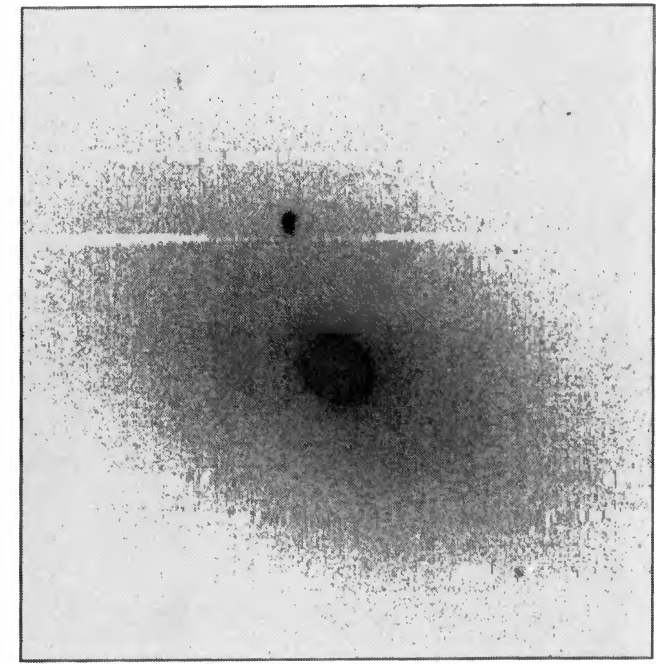

(a)

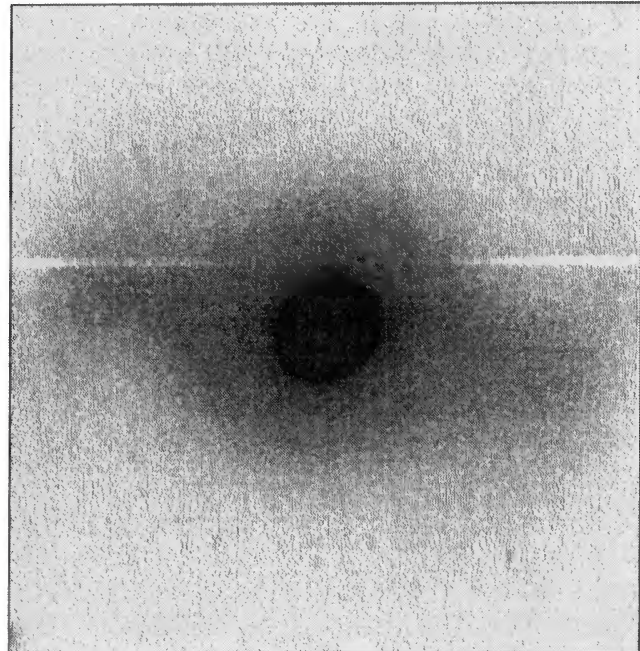

(b)

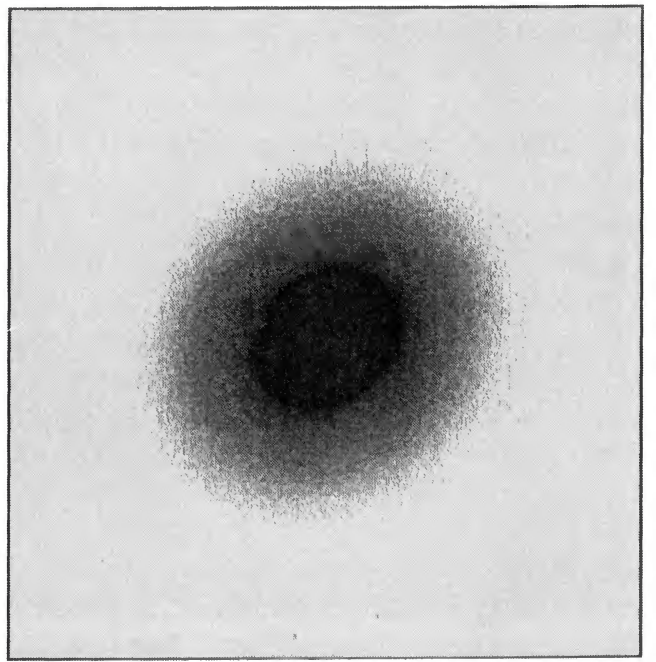

(c)

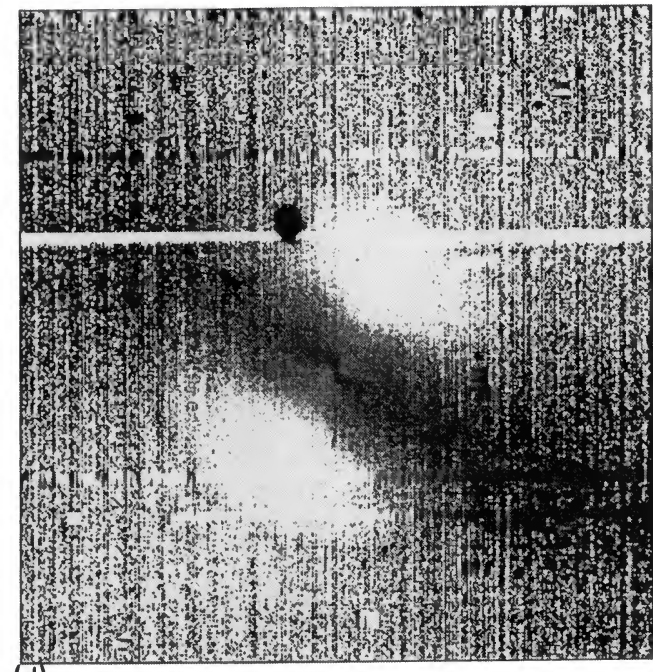

(d)

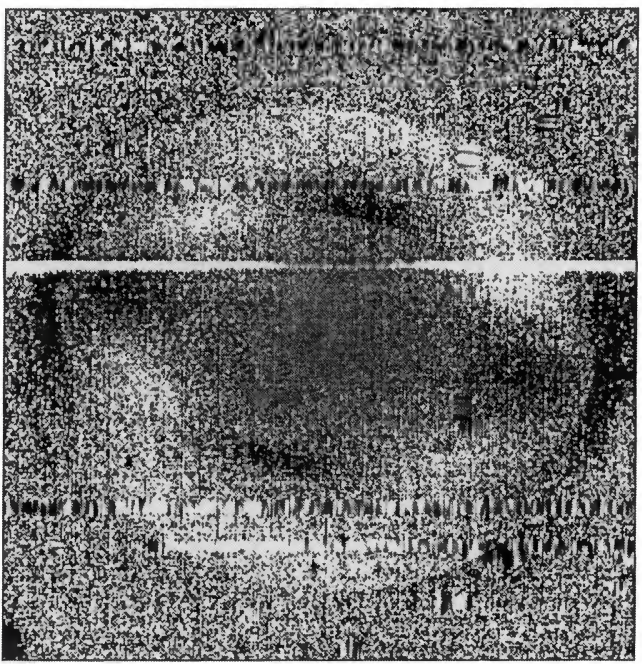

(e)

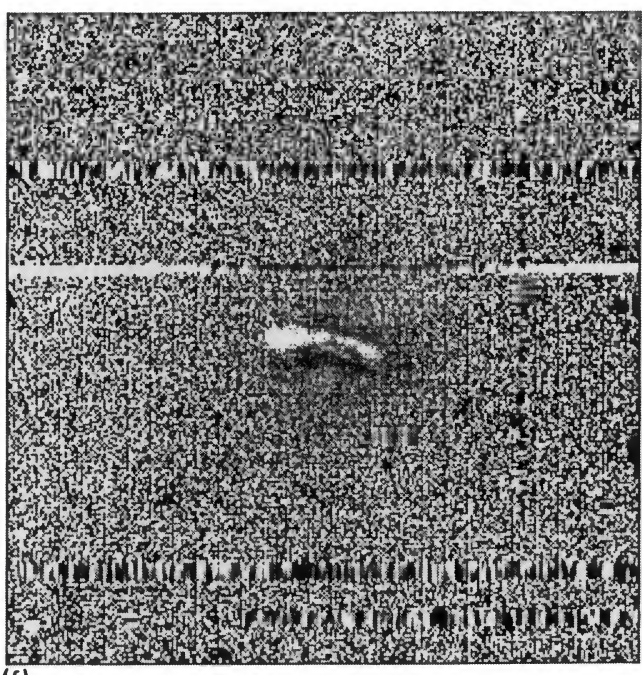

(f)

FIG. 2. Some examples of the image enhancement obtainable with division by a perfectly elliptical model image. North is at the top, east is at the left in all of the images. (a) Flattened GEC CCD image of NGC 584. The field is approximately $1.6 \times 1.6 \mathrm{arcmin}$. The white column near the center of the image (which is visible in almost all of the images) is due to several bad columns on the GEC CCD which have not been interpolated over. (These columns were ignored in the surface photometry reductions.) (b) Image of NGC 584 after division by a model image. The display range is from $20 \%$ below the mean to $20 \%$ above the mean. Note the very prominent disk running from the NE to the SW. The thin, tilted disk in the central regions may be an artifact. (c) Flattened GEC CCD image of an SB0 galaxy, NGC 936. The field of view is approximately $1.6 \times 1.6$ arcmin. Note that the bar is only faintly visible in this image. (d) Image of NGC 936 after division by a model image. Display range is $\pm 20 \%$. Here the bar is very prominent. (e) Flattened GEC CCD image of NGC 4374 , a wellknown dusty elliptical in the Virgo cluster. The dust can barely be seen as a notch in the isophotes nearly due east of the nucleus. The field of view is approximately $1.3 \times 1.3$ arcmin. (f) Image of NGC 4374 after division by a model image. Display range is $\pm 5 \%$. The dust appears to be in a ring around the nucleus.

(C) American Astronomical Society - Provided by the NASA Astrophysics Data System 


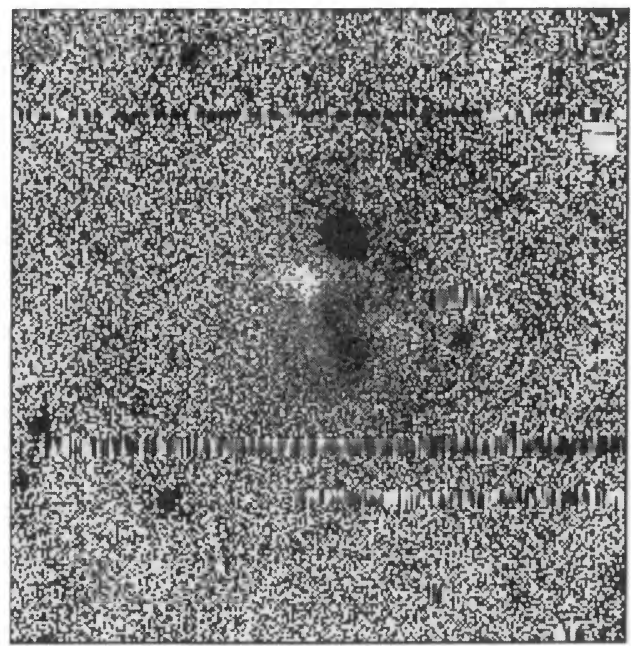

(a)

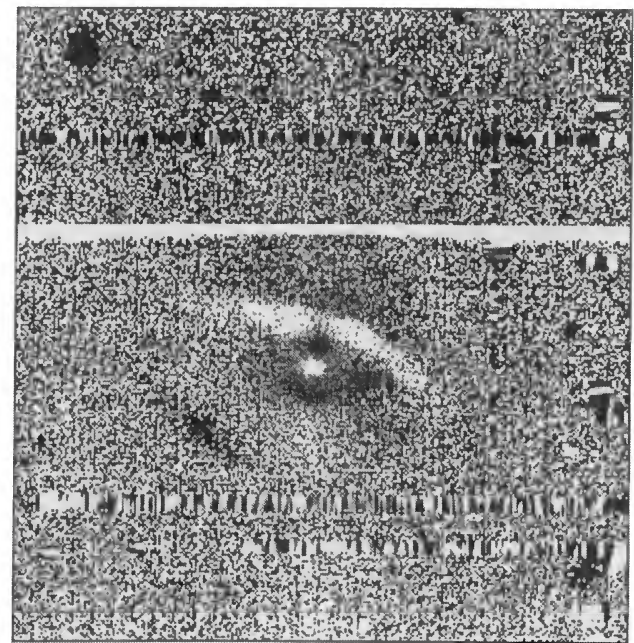

(b)

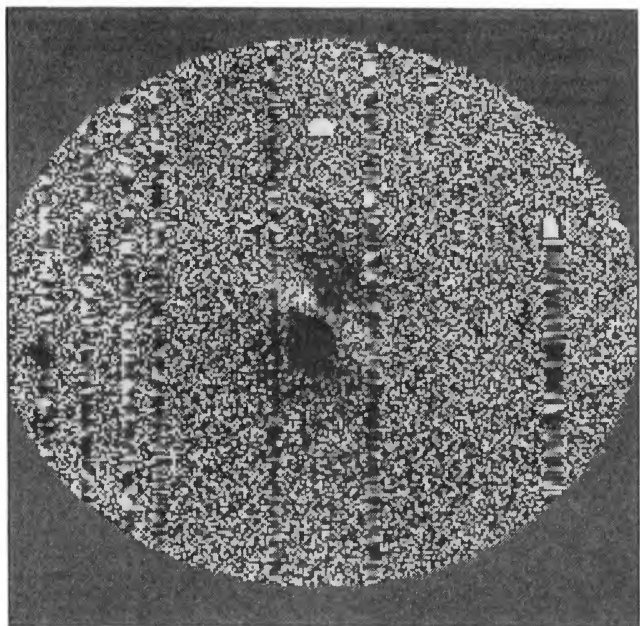

(c)

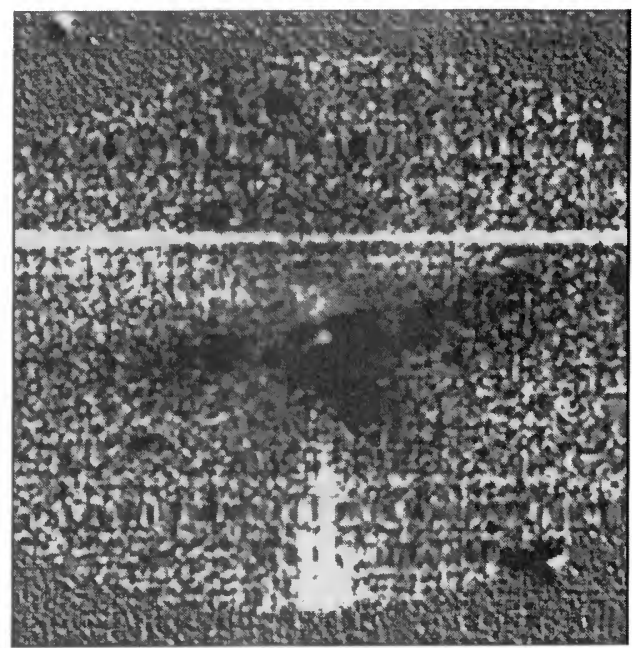

(d)

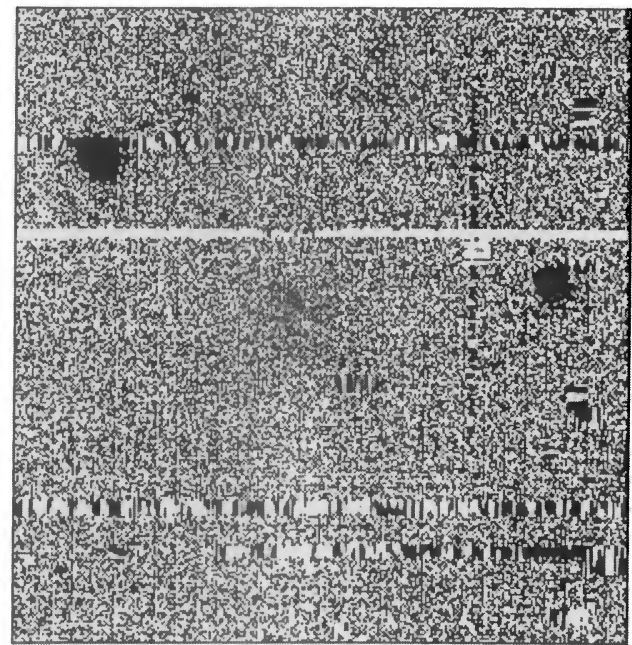

(e)

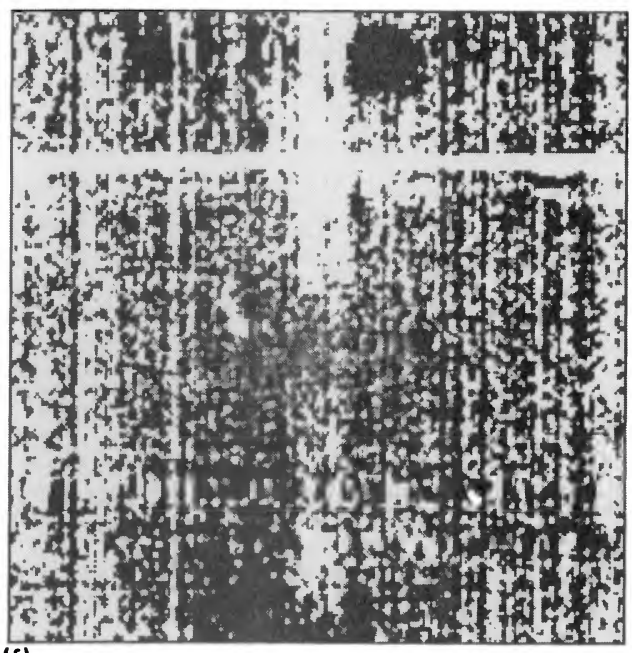

(f)

FIG. 3. Processed images of some of the galaxies discussed in Sec. III. North is at the left, east is at the bottom in all of the images. (a) Central regions of NGC 4278. The black dot to the left of center is a star superimposed near the nucleus of the galaxy. The white patch just SE of this star is a patch of dust near the nucleus. The nuclear regions appear to be broken up, possibly by much weaker dust. The field of view is approximately $1.1 \times 1.1$ arcmin; the display range is $\pm 5 \%$. (b) NGC 4660. This image has also been smoothed with a simple nearest-neighbor smoothing. A stellar disk is visible in the center of the image. The field of view is approximately $1.4 \times 1.4$ arcmin; the display range is $\pm 10 \%$. This disk is much weaker than that seen in NGC 584. (c) NGC 5485. The field of view is approximately $1.4 \times 1.4 \mathrm{arcmin}$; the display range is $\pm 10 \%$. The curved dust lane is very prominent. Note (1) the defect in the centering of the model image caused by the presence of the dust lane (which is manifested in the black and white "dipole" to the right of the dust lane), and (2) the black (i.e., bright) region to the SE of the nucleus. See the discussion in the text. (d) NGC 5638. The field of view is approximately $1.4 \times 1.4$ arcmin; the display range is $\pm 5 \%$. This is an example of a marginal detection or nondetection. (e) NGC 5831. The field of view is approximately $1.8 \times 1.8$ arcmin; the display range is $\pm 5 \%$. This image was taken with the TI CCD, unlike the other images in Figs. 2 and 3, which are from the GECCCD. (f) Central regions of NGC 5846. The field of view is approximately $1.1 \times 1.1$ arcmin; the display range is $\pm 5 \%$. The off-nuclear dust filament is visible as a white patch just to the left of the center of the image, between the two black "blobs" (companion galaxies). See the discussion in the text.

(c) American Astronomical Society • Provided by the NASA Astrophysics Data System 
known, and the galaxy has been classified as an elliptical in both RSA and RC2.

NGC 5485. A classical "dusty" elliptical, this galaxy was classified as a peculiar S0 by Sandage and Tammann (1981), presumably on the basis of the presence of a dust lane in an otherwise ordinary elliptical galaxy (Fig. 3(c)). The surfacephotometry parameters indicate, however, that the galaxy should be classified as an elliptical. In the absence of any contradictory kinematic data, we prefer this classification. Note the bright feature to the SE of the nucleus of the galaxy. It is not clear what this is. It may be a shell, or a piece of something that fell into the galaxy. It is probably real, because artificial features tend to be symmetric, and there is no counterpart to this feature on the opposite side of the nucleus.

NGC 5638. This galaxy was originally intended to illustrate a nondetection in a galaxy with good signal-to-noise. However, it is listed in Table I as possibly containing weak, diffuse dust, and there does seem to be a feature near the center of the galaxy in Fig. 3(d). In fact, we were unable to find a galaxy with good signal-to-noise that had absolutely no evidence of features. (An example of how bad the signalto-noise can be is given by NGC 5846; see below.) This illustrates the point that the nondetections really represent a lower limit to the number of ellipticals with features. We consider the nuclear feature in this object to be a marginal detection.

NGC 5831. This galaxy contains a fairly substantial dust lane which, like the dust lane in NGC 4374, is skewed with respect to the optical axes of the galaxy (Fig. 3(e)). This dust lane was previously observed by Schweizer (private communication). The galaxy is not a radio source $\left(S_{1.4 \mathrm{GHz}}<13\right.$ mJy; Hummel, Kotanyi, and Ekers 1983). The dust lane is not visible on conventional images of the galaxy.

NGC 5846. We confirm the weak, off-nuclear dust feature seen by Gallagher (1986). (Compare Fig. 3(f) with Gallagher's Fig. 1 (e); the black "blobs" in our image are companion galaxies which appear as black and white "blobs" in Gallagher's image.) Note that the dust is visible despite the relatively poor quality of the data. We would not have claimed this weak feature as a detection had it not been for Gallagher's independent detection of the same filament of dust. Gallagher also used a slightly different method in his work. Specifically, he made a "model" image by smoothing the original image, and then divided the original by the smoothed version. This technique is essentially analogous to ours. Gallagher had both $B$ and $V$ images of the galaxy, and the filament appears in both of them. As Gallagher points out, NGC 5846, like NGC 4374 and NGC 1316 (Fornax A), contains a hot, gaseous atmosphere detected in $\mathrm{x}$-ray observations and a cooler, ionized gas component, plus patches of dust which would seem to indicate the presence of a much cooler, neutral medium. (NGC 5846 has a weak nuclear radio source: Hummel, Kotanyi, and Ekers 1983.) As noted above, neither NGC 4374 nor NGC 1316 has detectable neutral hydrogen. It is unclear how the dust in these systems can survive in the presence of the very hot, $\mathrm{X}$-rayemitting gas, unless it resides in optically thick clouds. However, we note later that it seems that most of the dust we detect is in optically thin clouds, so it is not clear that this mechanism works either.

NGC 7052. This is another galaxy whose classification ( $E$ vs $\mathrm{S} 0$ ) is uncertain. It contains a narrow, extended radio source, which is at an angle of $\sim 50^{\circ}$ to the major axis of the galaxy. Gallagher (1986) reports that the dust lane is almost orthogonal to the galaxy major axis. Our processed image shows a weak quadrupole feature that is usually associated with the presence of a stellar disk. This would imply that the dust is in a polar orbit, and that the galaxy should probably be considered an S0. Note that galaxies with extended radio sources and genuine stellar disks are very rare, although in this particular instance the radio source is hardly a "classical" (Fanaroff-Riley Class II; Fanaroff and Riley 1974) double.

\section{DISCUSSION}

As noted above, our sample contains 116 elliptical galaxies, $33 \mathrm{~S} 0$ galaxies, and ten intermediate types. Some galaxies that might better be classified as "dusty ellipticals" are included among the S0s. Table II summarizes the statistics of the features found in the 159 galaxies for which usable images were available, broken down by Hubble type (E, E/SO, S0). Overall, slightly more than half $(\sim 53 \%)$ of the galaxies showed no signs whatsoever of any features at the level of the noise. For typical signal-to-noise values, this translates to upper limits of $\sim 3 \%$ contrast in the central regions of the galaxies, and somewhat larger limits ( $\sim 6 \%$ to $8 \%$ ) farther out. A rather large fraction (36\%) of the elliptical galaxies showed signs of dust absorption, mostly in small patches near the nucleus of the galaxy. Only three of the ellipticals (plus one "S0", NGC 5485, which we believe is really an elliptical) showed strong, large-scale dust lanes. Stellar disks were indicated in only ten galaxies classified as ellipticals; some of these had been previously reported (and one is probably a misclassified S0).

We found that 42 of the ellipticals ( $36 \%$ ) either definitely or very likely contain dust, either in patches typically found near the nucleus of the galaxy, or in well-defined lanes or rings. This is very similar to the results of other groups, e.g., Sadler and Gerhard (1985). Five of the transitional galaxies (50\%) also show possible or definite dust, as do $15(47 \%)$ of the S0s. More surprisingly, three of the elliptical galaxies (NGC 3610, NGC 4660, and NGC 4564) were found to definitely contain stellar disks, with seven more possible candidates. In all, approximately half of the elliptical galaxies

TABLE II. Statistics of features.

\begin{tabular}{lrrrr}
\hline \hline & E & E/S0 & S0 & Total \\
\hline Usable images: & 116 & 10 & 33 & 159 \\
& & & & \\
Featureless: & 61 & 4 & 5 & 70 \\
Possible dust: & 19 & 3 & 3 & 25 \\
Probable dust: & 8 & 0 & 3 & 11 \\
Definite dust: & 15 & 2 & 8 & 25 \\
Possible disk: & 7 & 0 & 2 & 9 \\
Definite disk: & 3 & 1 & 2 & 6 \\
Ambiguous: & 3 & 0 & 9 & 12 \\
Possible shells: & 3 & 0 & 2 & 5 \\
Other: & 1 & 0 & 3 & 4 \\
Boxy isophotes: & 2 & 1 & 3 & 6 \\
\hline \hline
\end{tabular}


showed "features" of some kind. Since the detection of any features is dependent on the signal-to-noise ratio of the data, these numbers represent lower limits. Indeed, many of the objects listed as having no features in Table I are galaxies for which the data were of relatively poor quality.

It is extremely difficult to quantify the strength of the features in the galaxies in a meaningful way that is not seriously misleading. The detectability of any given feature depends on both its size (large features are easier to find than small ones, both because large features cause greater perturbations in the surface photometry and because the human eye tends to connect the pieces of a large feature more readily) and its location in the image (the signal-to-noise ratio is a strong function of position in the image because the light of the galaxy is strongly peaked toward the center). In particular, it is much easier to find small patches of dust in the nuclear regions of a galaxy, where the signal-to-noise ratio is high, than in the outer regions of the galaxy. Indeed, in large features (such as the dust lane in NGC 5485), the signal-tonoise ratio varies across the feature, as one moves from the bright central regions with high $\mathrm{S} / \mathrm{N}$ to regions further out in the image where the $\mathrm{S} / \mathrm{N}$ is lower. Hence simply quoting numbers such as counts in the feature versus the "local" rms noise is essentially meaningless, since such values depend both on where the rms noise was measured and how large the feature is. Moreover, certain types of features are inferred by their effects on the residual image rather than by being directly observed. In particular, the quadrupole feature noted above is usually associated with the presence of a stellar disk, and galaxies showing this feature are reported in Table II as possible disk systems. This type of feature may also be caused by the presence of extremely boxy isophotes, as seems to be the case for NGC 7785. Less extreme but still boxy isophotes normally appear as a bright, $\mathrm{x}$-shaped "octupole" feature in the quotient image; only the very extreme cases seem likely to induce the instability that causes the quadrupole feature. The strength of these residuals is certainly related to the actual strength of the features that caused them, but it is not valid to say, for instance, that because the residual is $10 \%$ above the background, the actual feature is $10 \%$ above the background. This point is illustrated by NGC 584 (Fig. 2(b)): Note that the regions to the southeast and northwest of the center are white in the figure. This means that they are "low," i.e., that the model image is too high in these regions, while it is too low in the portion of the image occupied by the disk. Hence, the model in this case is really not a very good fit to the nondisk portion of the galaxy. Consequently, the flaring of the disk is almost certainly an artifact, and the amount of light in the residual is not a reliable measure of the amount of light actually in the disk. Nor is it obvious how one should go about constructing a better model with the existing data.

Some idea of the quantitative strength of the features may be obtained from the fact that the majority of the features detected were not seen unless the residual image was displayed with a contrast of $\pm 5 \%$. This implies that the detected features typically have relative amplitudes of a few percent. Some of the features are much stronger than this, and have been noted in the "Comments" column of Table I by indicating the contrast range at which they can be detected in our images. The small relative amplitude of the dips caused by the dust suggests that most of the dust found in ellipticals is optically thin, or is clumped into unresolved clouds. One could obtain better estimates of the extinction due to the dust by using blue and red images (as done by Sparks et al. 1985) to estimate the reddening of the back- ground light of the galaxy. Ideally, one would model the red image and (1) divide the blue image by the red model to make a color map, and (2) divide the red image by the red model to look for a residual at the location of any red features found in the color map, in order to confirm that the red features are indeed due to dust.

Figure 4 illustrates the completeness of our sample of ellipticals in terms of luminosity, and the luminosity distributions of galaxies with and without "features." The "completeness" is defined as the ratio of the number of galaxies in each magnitude bin that were examined in this search for features to the number of galaxies in the same magnitude bin in the original sample of Djorgovski (1985a). The error bars are Poissonian. We note that our survey sample is approximately uniformly complete at an $\sim 75 \%$ level for all magnitudes. The selection of galaxies in the original sample of Djorgovski (1985a) was based on apparent magnitudes and coordinates, so the completeness in luminosity is well understood (see that reference for details). Consequently, there should be no significant selection effects or hidden luminosity bias in the present sample. We find that the presence of dust is independent of luminosity, or for that matter, any other intrinsic characteristic of a galaxy; e.g., ellipticity, surface brightness, isophotal twist rate, etc. This supports the view that the dust is of an external origin; e.g., provided by mergers, unless the small nuclear dust patches are indeed ubiquitous in elliptical galaxies. The dusty galaxies do show a slight preference for lower-density environments, as indicated in Fig. 5. The "density" in this case is the number of luminous neighbors within a $2 \mathrm{Mpc}$ radius cylinder with $\Delta V<600 \mathrm{~km} \mathrm{~s}^{-1}$, correcting for distance effects with the selection function described by Davis and Huchra (1982). A Mann-Whitney rank-sum test shows that the difference between the mean local galaxy density for the sample of dusty galaxies and the sample of clean galaxies is significant at the $12 \%$ level, which is marginal. If the dust is acquired through mergers, this preference for low-density environments may simply be due to the fact that in higher-density environments the encounter velocities are sufficiently high that merging does not take place, or that the dust evaporates in the hot gaseous coronae that are often seen in clusters.

The galaxies that show definite or possible disks tend to have marginally lower luminosities than the sample as a whole (Fig. 4). They also have a very marginal tendency to be found in higher-density environments (Fig. 5). These systems may be bulges that never quite made it in developing their disks, perhaps because of tidal disruptions or sweeping. Not surprisingly, these galaxies also tend to have larger ellipticities than the sample as a whole, and have slightly larger two-wave Fourier residuals, as illustrated in Fig. 6; this was also noted by Carter (1987). The $P_{2}$ index in Fig. 6 is a median value of the two-wave Fourier residuals along the best-fit isophotes, rescaled by the scatter rms (see Djorgovski 1985a for details). (One of these galaxies, NGC 4564, is so flat that it may very well be a misclassified S0.) Unfortunately, the number of these systems in our survey is very small, so the statistical significance of these results is questionable. Nevertheless, these trends are intriguing, since they may provide us with some insights in answering an important question: why and how did some galaxies develop disks? And why did others not?

We found no correlation between the presence or absence of morphological features and any other observables available to us, particularly the parameters describing the radial and the azimuthal distribution of light, such as surface- 

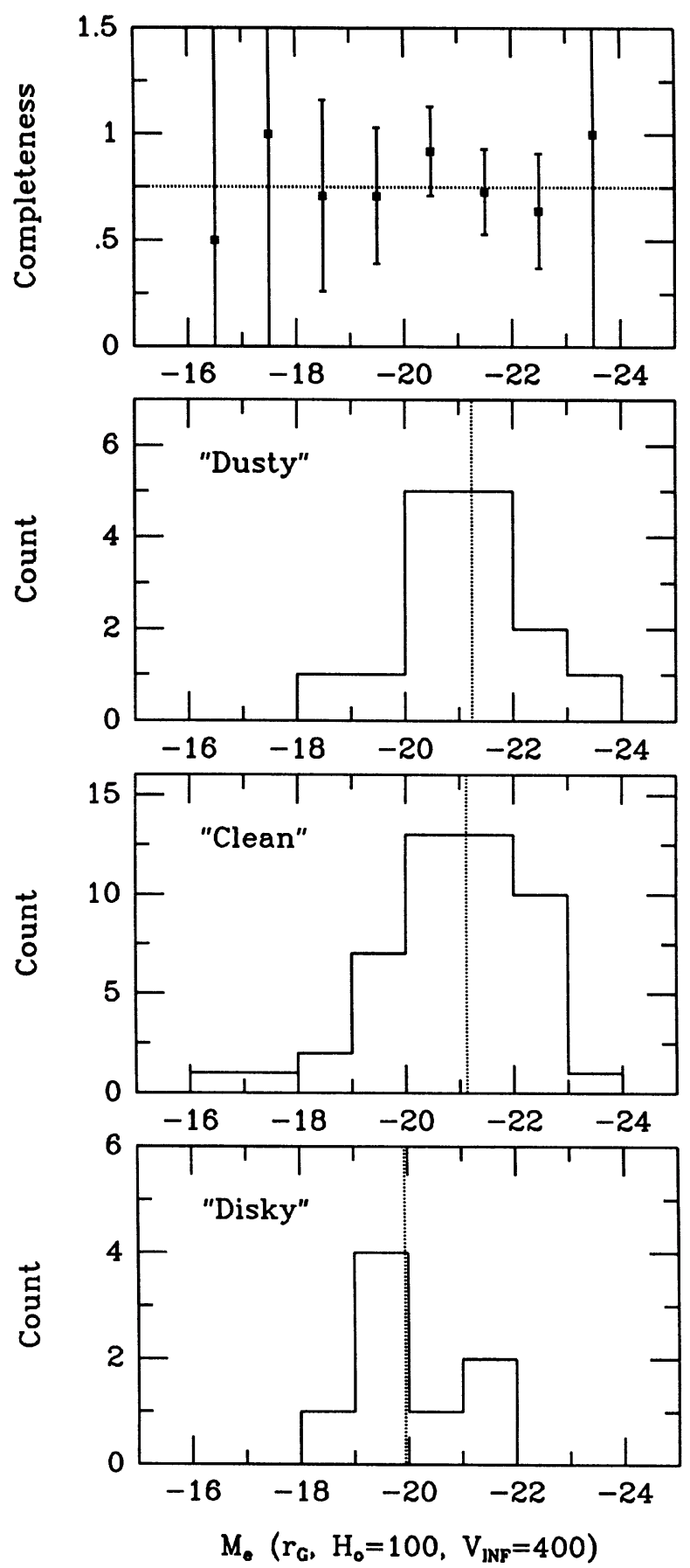

FIG. 4. Luminosity completeness of this survey, and luminosity distributions of three subsamples of ellipticals: those that show definite or probable dust ("dusty"), those with no dust detected, or with the dust unlikely ("clean"), and those with definite or possible stellar disks ("disky"). "Completeness" is defined as the fraction of galaxies in a given magnitude bin from the sample of Djorgovski (1985a) that were searched for features in the present work. The absolute magnitudes are as defined in Fig. 1 . Sample medians are indicated by the yertical dotted lines.

brightness slopes, ellipticity gradients, isophotal twist rates, etc. If mergers are responsible for the presence of dust, their disruptive influence has already been erased, at least at the radii that we probe, generally $\leqslant 2 r_{\mathrm{e}}$. It would be interesting to correlate the presence of dust in the inner parts of ellipti-
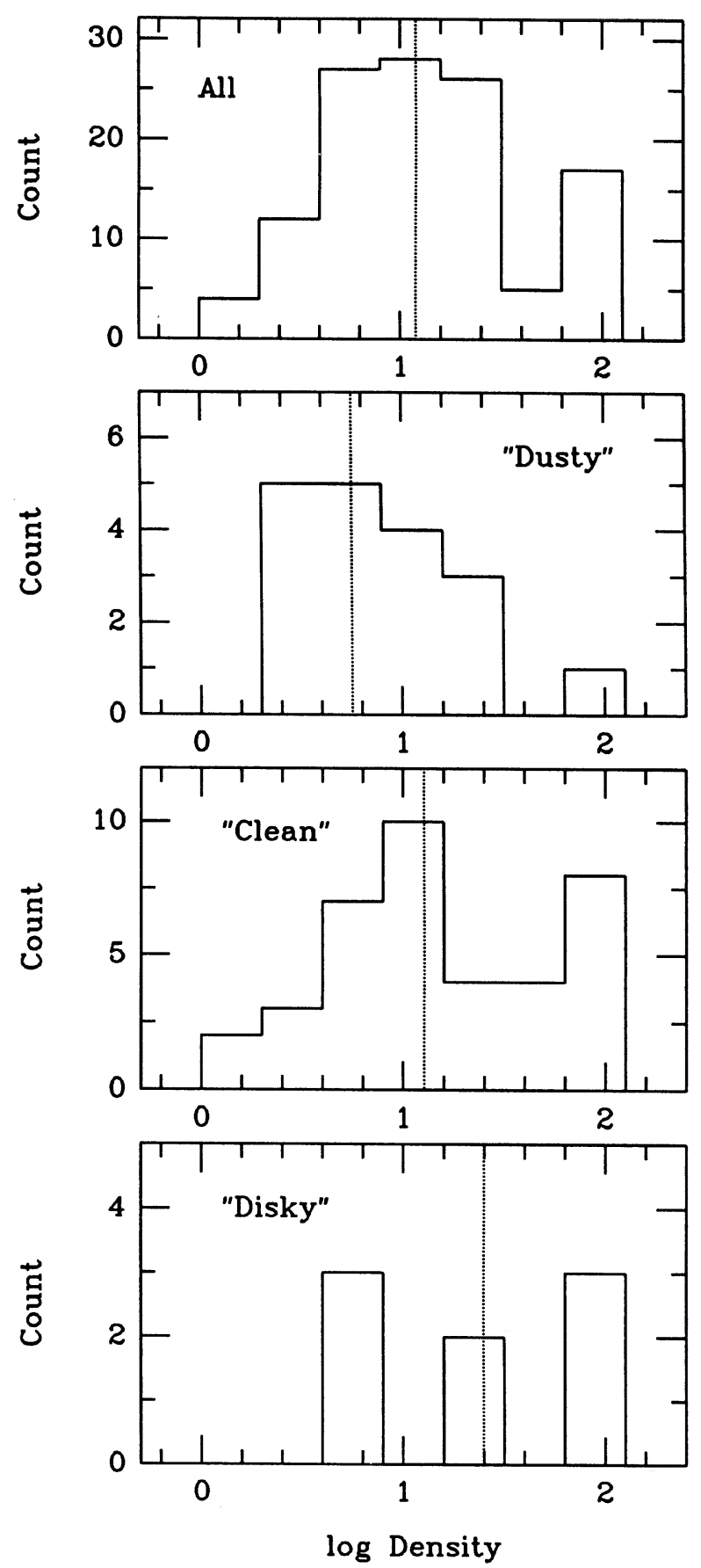

FIG. 5. Distributions of environmental densities for all galaxies in the sample (top), and the three subsamples as in Fig. 4. The density is defined as the extrapolated number of galaxies with $M_{B}<-17.9+5 \log h$ within a cylinder of radius $2 h^{-1} \mathrm{Mpc}$ with $\Delta V<600 \mathrm{~km} \mathrm{~s}^{-1}$, using the observed number density of galaxies from the complete CfA redshift survey sample. Sample medians are indicated by the vertical dotted lines.

cals with the presence of telling morphological features at larger radii (fainter isophotal levels), such as tidal tails, shells, etc. However, it has been suggested (Jura 1982, 1986) that all elliptical galaxies contain some dust, as a consequence of normal red giant evolution. If this is the case, one would expect to see a correlation between features such as 

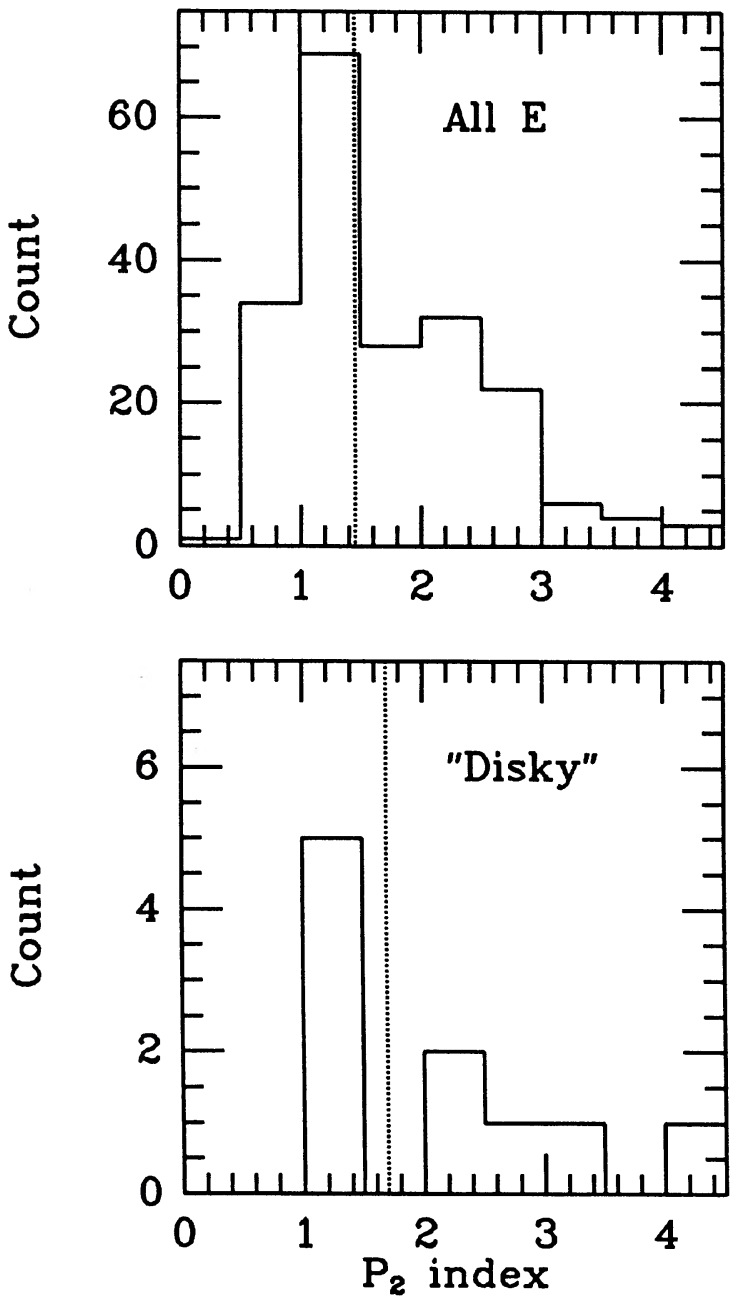

FIG. 6. Distributions of the $P_{2}$ index for all ellipticals in the sample (top), and those with definite or probable stellar disks (bottom). The $P_{2}$ index is a median value of the two-wave Fourier residuals along the best-fit isophotes, rescaled by the scatter rms (see Djorgovski 1985a for details). The presence of a disk superposed on a purely elliptical galaxy would produce such residuals, as observed. Sample medians are indicated by the vertical dotted lines.

tidal tails and shells and dust only if the dust is in a large, well-defined lane, since such dust lanes typically have angular-momentum distributions that cannot have been produced by dust and gas shed by stars in the galaxy (see, for example, Ebneter and Balick 1985, and references therein).

Finally, we examined the behavior of ellipticals with and without features in the context of the "fundamental plane" of elliptical galaxies (Djorgovski and Davis 1987; Djorgovski 1987b; Dressler et al. 1987), as illustrated in Fig. 7. In Fig. 7, the projected central velocity dispersion $\sigma_{0}$, measured in $\mathrm{km} \mathrm{s}^{-1}$, is plotted against the mean surface brightness within the $r_{\mathrm{e}}$ isophote, $\left\langle\mu_{\mathrm{e}}\right\rangle$, measured in $r_{G}$ magnitudes per square arcsecond. $r_{G}$ is the Spinrad red CCD bandpass with the GEC CCD, as defined by Djorgovski (1985b). This is an interesting check, since there could, in principle, be significant trends in the multidimensional parameter space of observables which would not be apparent when projected, e.g., on the luminosity axis. Unfortunately, no such trends were found. The locus occupied by galaxies in the fundamental plane of ellipticals probably reflects the processes by which the galaxies formed. The lack of any correlations between the presence or absence of dust and the parameters that determine the fundamental plane again may simply suggest that some dust is present as a normal component in all ellipticals. We also note a lack of correlation between surface brightness and the presence of dust: For all practical photometric purposes, obscuration by dust in elliptical galaxies is negligible.

\section{CONCLUSIONS}

The presence of relatively low-surface-brightness features in almost half of the elliptical galaxies studied here demonstrates that elliptical galaxies are not the featureless objects they were once thought to be. The occurrence of weak stellar disks in some of these objects also shows that the morphological distinction between elliptical and S0 galaxies is somewhat blurred. Detailed kinematic studies are not available for many of these galaxies, so it is impossible to say whether there is a significant dynamical difference between the two categories. These results emphasize the need for a physical rather than morphological classification of early-type galaxies. However, even kinematical measurements may not be enough: Dressler and Sandage (1983) discovered a class of slowly rotating S0 galaxies, which they call "diskless S0s." It is tempting to pinpoint the source of dynamical support in flattened systems (rotation versus the anisotropic velocity dispersion tensor) as the main physical discriminant between the "true ellipticals" and SOs. However, both rotation and anisotropic velocity dispersions are present, and their relative dominance is a function of luminosity (Davies et al. 1983). The ellipticals and S0s are two different classes of galaxies, but the boundary between them is not sharp.

A large fraction of the ellipticals show evidence for the presence of dust in patches, but only a relatively small number show sizable dust lanes. Since the prominent dust lanes are seen clearly only when they are nearly edge-on (the dust has relatively low optical depths), it is not clear whether or not the ellipticals with patchy dust also possess such lanes. Sadler and Gerhard (1985) have estimated that $\sim 40 \%$ of all elliptical galaxies contain large-scale dust structures, based on the fraction of galaxies with dust lanes that they detected in their sample and statistical arguments about the orientations of the galaxies to our line of sight. This is surprisingly (and probably coincidentally) close to the fraction of elliptical galaxies in our sample in which we detect dust in any configuration. If this result is more than a coincidence, it might imply that the galaxies that contain patchy dust are really dust-lane galaxies seen at an unfavorable orientation. However, we cannot rule out the possibility that small nuclear dust patches are an ubiquitous and normal component of elliptical galaxies.

The origin of these features is not clear. The large dust lanes are often supposed to be remnants of mergers or cannibalism. It is not obvious that mergers are necessary to produce the very common nuclear dust patches, unless these are only the visible portion of a much larger (but relatively thin) dust lane which is rendered invisible by its orientation. The origin of the stellar disks is even more obscure: The galaxies could be flattened systems which happen to have extremely large, dominant bulges (and therefore are related more closely to the true SOs), or they could be elliptical systems in which a rotating disk of gas acquired in a merger has transformed a large fraction of its gas into stars, which will remain 


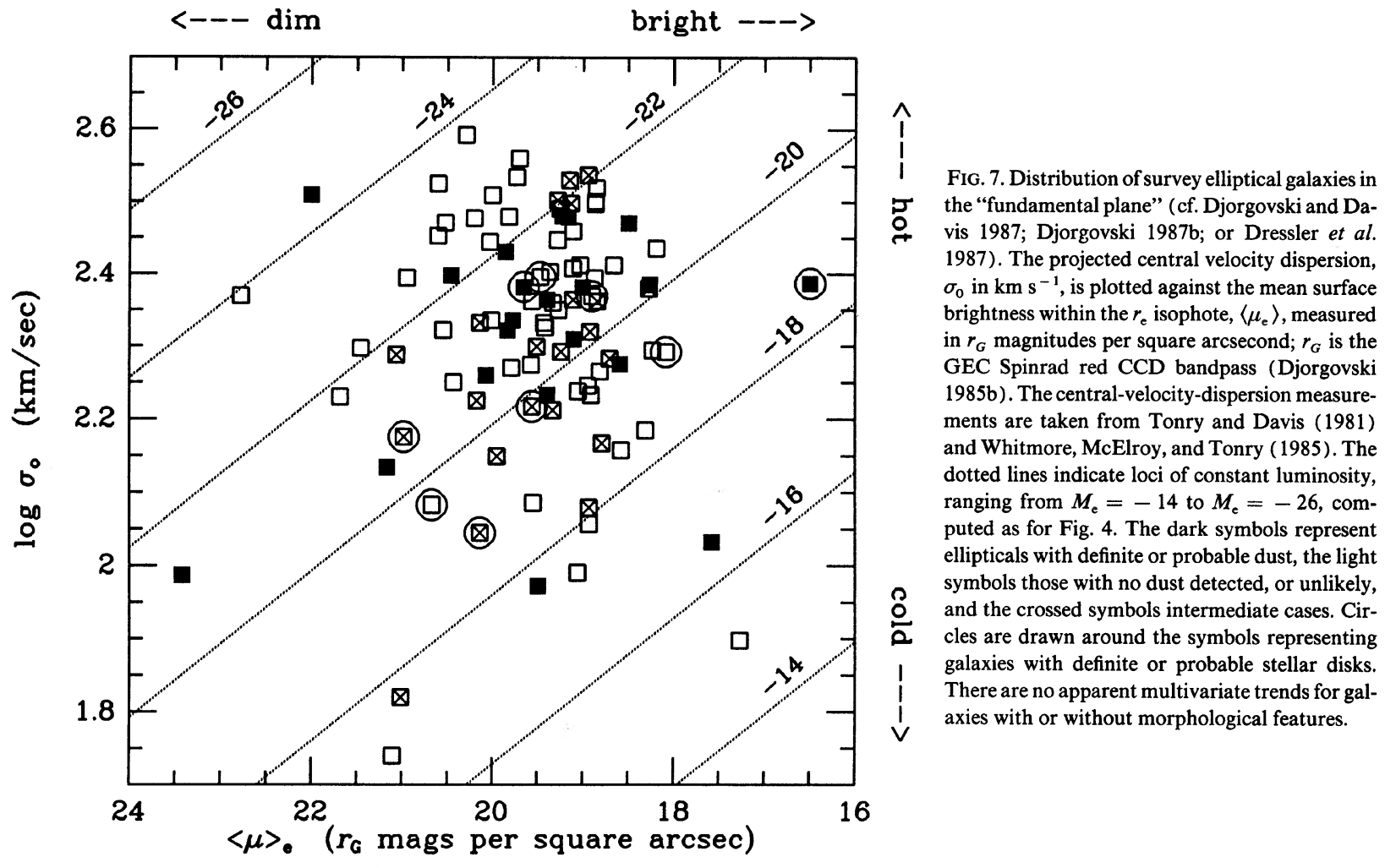

in the orbits in which they were formed. Since numerous ellipticals with large dust features do show evidence for star formation in the dust lanes (Brosch 1987; Ebneter and Balick 1983) it seems clear that such a process could happen. It is more likely to explain the smaller disks seen in galaxies like NGC 4660 than the very large (and massive?) stellar disk seen in NGC 584. Explaining why some ellipticals, presently known as "bulges," did acquire or develop stellar disks, and some did not, is one of the central problems in our understanding of galaxy formation. Transitional cases such as those described in this paper may provide one of the leads.
This work was supported in part by the NSF grant AST8614552 . We would like to thank the staff of Lick Observatory for their help in obtaining the data used in this work, Tod Lauer for communicating his model-image-generating subroutine to us, Tom Statler for numerous illuminating discussions, and Rosie Wyse for useful comments on the manuscript and general moral support. K. E. acknowledges the support of an Amelia Earhart Fellowship from Zonta International, and a Graduate Opportunity Fellowship from the University of California. S. D. acknowledges partial support from Harvard University.

\section{REFERENCES}

Brosch, N. (1987). Mon. Not. R. Astron. Soc. 225, 257.

Carter, D. (1987). Astrophys. J. 312, 514.

Davies, R., Efstathiou, G., Fall, S. M., Illingworth, G., and Schechter, P. (1983). Astrophys. J. 266, 41

Davies, R., and Illingworth, G. (1983). Astrophys. J. 266, 516.

Davis, M., and Huchra, J. (1982). Astrophys. J. 254, 437.

de Vaucouleurs, G., de Vaucouleurs, A., and Corwin, H. G. (1976). Second

Reference Catalog of Bright Galaxies (University of Texas, Austin) (RC2).

Djorgovski, S. (1985a). Ph.D. thesis, University of California, Berkeley.

Djorgovski, S. (1985b). Publ. Astron. Soc. Pac. 97, 1119.

Djorgovski, S. (1986). Proc. SPIE 627, 674.

Djorgovski, S. (1987a). In Globular Clusier Systems in Galaxies, IAU Symposium No. 126, edited by J. Grindlay and A. G. D. Philip (Reidel, Dordrecht) (in press)

Djorgovski, S. (1987b). In Structure and Dynamics of Elliptical Galaxies, IAU Symposium No. 127, edited by T. de Zeeuw (Reidel, Dordrecht) (in press)

Djorgovski, S., and Davis, M. (1987). Astrophys. J. 313, 59.
Djorgovski, S., and Ebneter, K. (1986). In Instrumentation and Research Programmes for Small Telescopes, IAU Symposium No. 116, edited by J. B. Hearnshaw and P. L. Cottrell (Reidel, Dordrecht), p. 277.

Djorgovski, S., Spinrad, H., Pedelty, J., Rudnick, L., and Stockton, A. (1987). Astron. J. 93, 1307.

Dressler, A., Lynden-Bell, D., Burstein, D., Davies, R. L., Faber, S. M., Terlevich, R. J., and Wegner, G. (1987). Astrophys. J. 313, 42.

Dressler, A., and Sandage, A. (1983). Astrophys. J. 265, 664.

Ebneter, K., and Balick, B. (1983). Publ. Astron. Soc. Pac. 95, 675.

Ebneter, K., and Balick, B. (1985). Astron. J. 90, 183.

Faber, S. M., Balick, B., Gallagher, J. S., and Knapp, G. R. (1978). Astrophys. J. 214, 383.

Fanaroff, B. L., and Riley, J. M. (1974). Mon. Not. R. Astron. Soc. 167, $31 \mathrm{p}$.

Forman, W., Jones, C., and Tucker, W. (1985). Astrophys. J. 293, 102.

Gallagher III, J. S. (1986). Publ. Astron. Soc. Pac. 98, 81.

Gunn, J. E. (1978). In Active Galactic Nuclei, edited by C. Hazard and S. Mitton (Cambridge University, Cambridge), p. 213.

Hawarden, T. G., Elson, R. A. W., Longmore, A. J., Tritton, S. B., and 
Corwin, H. G., Jr. (1981). Mon. Not. R. Astron. Soc. 196, 747.

Heckman, T. M., Smith, E. P., Baum, S., van Breugel, W. J. M., Miley, G.

K., Illingworth, G. D., Bothun, G. D., and Balick, B. (1986). Astrophys. J. 311, 526.

Hubble, E. (1930). Astrophys. J. 71, 231

Huchra, J. P., Davis, M., Latham, D., and Tonry, J. (1983). Astrophys. J. Suppl. 52, 89.

Hummel, E., Kotanyi, C. G., and Ekers, R. D. (1983). Astron. Astrophys. $127,205$.

Jura, M. (1982). Astrophys. J. 254, 70.

Jura, M. (1986). Astrophys. J. 306, 483.

Knapp, G. R., Kerr, F. J., and Williams, B. (1978). Astrophys. J. 222, 800.

Knapp, G. R., Turner, E. L., and Cunniffe, P. E. (1985). Astron. J. 90, 454.

Kormendy, J. (1983). Astrophys. J. 275, 529.

Kotanyi, C. G., and Ekers, R. D. (1979). Astron. Astrophys. 73, L1

Lauer, T. R. (1985a). Astrophys. J. Suppl. 57, 473.

Lauer, T. R. (1985b). Mon. Not. R. Astron. Soc. 216, 429.

Merritt, D., and de Zeeuw, T. (1983). Astrophys. J. Lett. 267, L19.
Nilson, P. (1973). Uppsala General Catalog of Galaxies, Uppsala Astron. Obs. Ann. 6 (UGC).

Sadler, E. M., and Gerhard, O. E. (1985). Mon. Not. R. Astron. Soc. 214, 177.

Sandage, A., and Tammann, G. A. (1981). A Revised Shapley-Ames Catalog of Bright Galaxies (Carnegie Institution of Washington, Washington, DC) (RSA).

Sparks, W. B., Wall, J. V., Thorne, D. J., Jorden, P. R., van Breda, I. G., Rudd, P. J., and Jorgensen, H. E. (1985). Mon. Not. R. Astron. Soc. 217, 87.

Stover, R., Lauer, T., Terndrup, D., Goodrich, R., and De Robertis, M. (1985). VISTA Manual, Version 3 (Lick Observatory, University of California, Santa Cruz)

Tonry, J., and Davis, M. (1981). Astrophys. J. 246, 666.

Wade, C. M. (1960). Observatory 80, 235.

Whitmore, B., McElroy, D., and Tonry, J. (1985). Astrophys. J. Suppl. 59, 1. 